\title{
WEIGHTED NONLINEAR POTENTIAL THEORY
}

\author{
DAVID R. ADAMS
}

\begin{abstract}
The potential theoretic idea of the "thinness of a set at a given point" is extended to the weighted nonlinear potential theoretic setting - the weights representing in general singularities/degeneracies-and conditions on these weights are given that guarantee when two such notions are equivalent at the given point. When applied to questions of boundary regularity for solutions to (degenerate) elliptic second-order partial differential equations in bounded domains, this result relates the boundary Wiener criterion for one operator to that of another, and in the linear case gives conditions for boundary regular points to be the same for various operators. The methods also yield two weight norm inequalities for Riesz potentials

$$
\left(\int\left(I_{\alpha} * f\right)^{q} v d x\right)^{1 / q} \leqslant c\left(\int f^{p} w d x\right)^{1 / p},
$$

$1<p \leqslant q<\infty$, which at least in the first-order case $(\alpha=1)$ have found some use in a number of places in analysis.
\end{abstract}

1. Introduction. In recent years a number of papers have appeared pointing out the connection between the theory of weights a la Muckenhoupt-Wheeden-FeffermanCoifman et al. and the pointwise behavior of solutions to certain elliptic second order partial differential equations in bounded domains of Euclidean $N$-space. In particular, and especially of interest to us in this paper, is the question of the boundary regularity of such solutions to Dirichlet problems for certain degenerate divergence form linear and nonlinear operators as well as for linear nondivergence uniformly elliptic operators. See $[16,34,9]$. The condition that must be met at the boundary is, of course, some analogue of the Wiener criteria. These criteria can be expressed as a Dini type integral involving a capacity set function built out of the differential operator. In the simplest case, these capacities can be viewed as a slight modification of the Newtonian capacity (associated with the Laplace operator) using the structure coefficients of the differential operator. In the nondivergence linear equation case, these capacities may well involve the use of the Green's function for the differential operator on some fixed ball. However, in any case the idea is to study these capacities and their corresponding Wiener criteria in order to obtain useful sufficient conditions that will imply when two differential operators have the same boundary regular points.

Received by the editors May 9, 1985. Parts of this paper were presented at the AMS meeting in Minneapolis, Minnesota, November 3, 1985; entitled Some weighted estimates for potentials (814-31-67). 1980 Mathematics Subject Classification (1985 Revision). Primary 31B25, 31C15, 46E35, 26D10.

Key words and phrases. Capacity, thinness, two-weight embeddings, boundary regularity. 
First of all, this concept of boundary regularity is replaced by the equivalent potential theoretic idea of thinness - in that we concentrate on potentials of measures both in the linear as well as the nonlinear cases. Consequently, this necessitates the introduction of nonlinear potentials based on these degenerate operators. In the nondegenerate case, this theory is due to $[\mathbf{2 6}, \mathbf{2 5}, \mathbf{7}, \mathbf{2 1}$, and 20]. Roughly speaking, the idea is that a boundary point is regular for a domain and a given differential operator, if the complement of that domain is not thin at the point for a specific weight function (which, as bêfore, comes from the differential operator). See $\S 8$ for the definitions and especially Examples 8.5(e) and (f). These examples illustrate, at least in the linear case $(p=2)$, what this paper sets out to achieve. In this regard, our results are Theorem 8.2 and Corollary 8.3.

As a by-product of this investigation, we get, by the same techniques, certain imbedding theorems for Riesz potentials on weighted $L^{p}$-spaces. Results of this type have received considerable attention lately. See e.g. $[34,18,16,13]$. The interesting thing is that our technique basically returns to the methods established in the early 1970s for treating the one-side weight case as opposed to the now "two-weight" case. The key to this new-found vigor is the work of T. Wolff [20]. Our result is Theorem 7.1 (and Corollary 7.7), for $q>p$. In Theorem 7.3, we also give a version of a result of C. Fefferman [17] when $q=p$. Our result also extends work of [12 and 13].

Although our results rest on the basic estimates of $T$. Wolff, the principal technique that makes the results of this paper valid is the knowledge of the weighted capacity of a ball in $N$-space. See $\S 6$. Another result that might be of some independent interest is the capacitary strong-type inequality obtained in $\$ 5$. It too relies in a significant way on the estimates originally due to T. Wolff.

§2. Preliminaries about weights are discussed.

§3. Energy and nonlinear potentials are introduced on the "weighted spaces." It is here that the analogue of Wolff's inequality is discussed.

$\S 4$. Weighted Riesz capacity is defined and several alternative formulations are given; see especially (4.9) which is apparently not known even in the classical (or $p=2, \alpha=1)$ case.

$\S 5$. The weighted capacitary strong-type inequality is derived as a consequence of Wolff's inequality and the boundedness principle in nonlinear potential theory.

$\S 6$. Weighted Riesz capacity of a ball in $N$-space is calculated.

§7. The two-weight imbeddings for Riesz potentials are derived.

$\S 8$. Weighted thinness and its dependence on the weight are discussed. Examples illustrate the relationship to second-order elliptic partial differential equations.

\section{Preliminaries.}

2.1. Weights. For all of what follows in this subsection, the reader is referred to the standard references on weights; see esp. [28, 29, 14, 23]. A nonnegative Lebesgue measurable function $w(x)$ on $\mathbf{R}^{N}$ is an $A_{p}$ weight (or simply $w \in A_{p}$ ) if

$$
\sup _{Q}\left(f_{Q} w d x\right)\left(f_{Q} w^{-1 / p-1} d x\right)^{p-1}<\infty
$$


where the supremum is taken over all coordinate cubes $Q$ in $\mathbf{R}^{N} . w$ is an $A_{1}$ weight if

$$
\sup _{Q}\left(f_{Q} w d x\right)\left(\inf _{x \in Q} w(x)\right)^{-1}<\infty
$$

and an $A_{\infty}$ weight if

$$
\sup _{Q}\left(f_{Q} w d x\right)\left(\exp f_{Q} \log w^{-1} d x\right)<\infty .
$$

Here $f_{Q} w d x$ denotes the usual integral average of $w$ over $Q$. It is well known that $A_{\infty}$ can also be characterized by $A_{\infty}=\cup_{p<\infty} A_{p}$ or by $w \in A_{\infty}$ iff there are positive constants $c_{i}$ and $\delta_{i}, i=1,2$, such that for any coordinate cube $Q$ and any measurable subset $E \subset Q$,

$$
c_{1}\left(\frac{|E|}{|Q|}\right)^{\delta_{1}} \leqslant \frac{w(E)}{w(Q)} \leqslant c_{2}\left(\frac{|E|}{|Q|}\right)^{\delta_{2}} .
$$

Here $w(E)=\int_{E} w d x$ and $|E|=\int_{E} d x$, with, as usual, $d x$ denoting Lebesgue measure on $\mathbf{R}^{N}$. Since the constants in (2.4) are independent of the sets, it is clear that $\delta_{1} \geqslant 1$ and $\delta_{2} \leqslant 1$.

A weight $w$ is said to be a doubling weight if there is a constant $c_{0}$ such that for all coordinate cubes $Q, w\left(Q^{*}\right) \leqslant c_{0} \cdot w(Q)$ where $Q^{*}$ is the cube with the same center as $Q$ but with twice the edge length-the double of $Q$. It is well known that $A_{\infty}$ weights are doubling weights.

Most of the weights considered in this paper will be either $A_{p}$ weights or weights satisfying

$$
w^{-1 / p-1} \in A_{\infty} .
$$

Note that if $w \in A_{p}$ then (2.5) holds.

For later considerations, we now introduce a local dimension number associated with a weight $w$ satisfying (2.5). For fixed $p$, set $d_{w}(x)=\inf d$ where the infimum is over all $d$ satisfying

$$
\sup _{0<r \leqslant 1} r^{d}\left(f_{B(x, r)} w^{-1 / p-1}\right)^{1-p}<\infty .
$$

Here $B(x, r)$ denotes the ball $\{y:|x-y|<r\}$ in $\mathbf{R}^{N}$. Note that (2.4) implies $d_{w}(x) \leqslant N\left(\delta_{1}-1\right)(p-1)$ for all $x$. Of course $d_{w}(x)=0$ a.e. $x$. For $w(x)=|x|^{\beta-N}$, $\beta<N p, d_{w}(0)=N-\beta$.

2.2. Potentials and capacity. The Riesz potential of order $\alpha, 0<\alpha<N$, of a Borel measure $\mu$ on $\mathbf{R}^{N}$ is $I_{\alpha} * \mu(x)=\int|x-y|^{\alpha-N} d \mu(y)$. The fractional maximal function of order $\alpha$ of $\mu$ is $M_{\alpha} \mu(x)=\sup _{r>0} r^{\alpha-N} \mu(B(x, r))$. Recall that $M_{0} \mu$ is the classical Hardy-Littlewood maximal function of $\mu$. If $d \mu=w d x$, we simply write $I_{\alpha} * w$ and $M_{\alpha} w$, respectively. Similarly, we shall denote the class of all $p$ th power summable functions on $\mathbf{R}^{N}$ with respect to the measure $\mu$, by $L^{p}(\mu)$, and its norm by $\|\cdot\|_{L^{p}(\mu)}$. And when $d \mu=w d x$, we use the letter $w$ in place of $\mu$. Recall that $M_{0}$ and the various Caldéron-Zygmund singular integrals are bounded operators on $L^{p}(w)$ when $w \in A_{p}, 1<p<\infty$. 
The weighted Riesz capacity to be studied extensively in this paper, under the assumption (2.5) on the weight, can be defined by

$$
R_{\alpha, p}^{w}(E)=\inf \left\{\int f(x)^{p} w(x) d x: f \geqslant 0 \& I_{\alpha} * f \geqslant 1 \text { on } E\right\}
$$

for an arbitrary set $E$. In $\S 4$, we will give alternate formulations of $R_{\alpha, p}^{w}$; see esp. (4.9) which even in the unweighted case is new. For the unweighted case, see esp. [26, 25].

2.3. Finally, we shall denote the support of a measure $\mu$ by $\operatorname{supp} \mu$, and say that two positive quantities $A$ and $B$ are comparable (written $A \sim B$ ) if the ratio $A / B$ is bounded above and below by positive finite constants independent of the variables of $A$ and $B$ under study. And the letter $c$ will denote a generic constant, independent of said (or implied) variables: $A \sim B$ iff $c^{\prime} B \leqslant A \leqslant c B$.

3. Energy and nonlinear potentials. Let $w$ be a weight satisfying (2.5) and $\mu$ a Borel measure on $\mathbf{R}^{N}$, then the ( $\left.\alpha, p\right)$-energy of $\mu$ with weight $w$ is defined to be

$$
\mathscr{E}_{\alpha, p ; w}(\mu)=\int_{\mathbf{R}^{N}}\left[I_{\alpha} * \mu(x)\right]^{p^{\prime}} w(x)^{-1 / p-1} d x
$$

$p^{\prime}=p / p-1$. Note that if we write $\left(I_{\alpha} * \mu\right)^{p^{\prime}}=\left(I_{\alpha} * \mu\right)^{1 / p-1} \cdot I_{\alpha} * \mu$ and interchange the order of integration in (3.1), we get

$$
\mathscr{E}_{\alpha, p ; w}(\mu)=\int U_{\alpha, p ; w}^{\mu}(x) d \mu(x)
$$

where

$$
U_{\alpha, p ; w}^{\mu}(x)=I_{\alpha} *\left(w^{-1} \cdot I_{\alpha} * \mu\right)^{1 / p-1}(x)
$$

is the so-called weighted "nonlinear potential" of the measure $\mu$, with weight $w$. When $w \equiv 1$, these nonlinear potentials have been studied extensively by several authors during the last decade; see esp. $[25,26,7,21,3,20]$. In fact, the pointwise behavior of these unweighted nonlinear potentials has added to our understanding of the local properties of Sobolev functions and solutions to certain elliptic (nonlinear) differential equations, at least for those values of $p>2-\alpha / N$. Notice that the classical case $\alpha=1, p=2, N=3$ falls into this range; in fact, $U_{1,2 ; 1}^{\mu}(x)$ is just a constant multiple of the Newtonian potential $I_{2} * \mu(x)$. The reason for this limitation in the values of $p$ is that these unweighted nonlinear potentials are identically infinite when the measure $\mu$ is a Dirac measure and $p \leqslant 2-\alpha / N$. This difficulty illustrates one of the problems that was encountered when a nonlinear potential theory was being developed in analogy to the classical $(p=2)$ or Hilbert space theory. A way around this difficulty is to instead deal directly with the energy itself rather than to try to estimate $U$, and then to eventually replace it by some simpler equivalent expression. This we do below in Theorem 3.2. This approach is due to T. Wolff [20]. Wolff's argument was simplified first by J. L. Lewis and then later by P. Nilsson. The present argument uses the earlier work of Muckenhoupt-Wheeden [30], 
but in fact is close to the spirit of Lewis' proof. The result we need is

Proposition 3.1. If $w \in A_{\infty}, 1<p<\infty$, and $0<\alpha<N$, then there is a constant $c$ depending only on $\alpha, p$ and the $A_{\infty}$ constant such that

$$
\int\left(I_{\alpha} * \mu\right)^{p} w d x \leqslant c \int\left(M_{\alpha} \mu\right)^{p} w d x .
$$

THEOREM 3.2. If $w$ satisfies (2.5), then

$$
\mathscr{E}_{\alpha, p ; w}(\mu) \sim \int W_{\alpha, p ; w}^{\mu}(x) d \mu(x)
$$

where

$$
W_{\alpha, p ; w}^{\mu}(x)=\int_{0}^{\infty}\left[t^{\alpha p-N} \mu(B(x, t))\right]^{1 / p-1}\left(f_{B(x, t)} w^{-1 / p-1}\right) \frac{d t}{t}
$$

This result is essentially the key to much of the rest of this paper. Notice that $W_{\alpha, p ; 1}^{\mu}$ is finite except at the point of concentration of a Dirac measure $\mu$ when $1<p<N / \alpha$. And, in fact, $U_{\alpha, p ; 1}^{\mu}$ and $W_{\alpha, p ; 1}^{\mu}$ are comparable when $p>2-\alpha / N$; see [3]. However, we now are bypassing the need for the $U$-potentials!

Proof of Theorem 3.2. Applying Proposition 3.1 to the energy together with the simple inequality

$$
M_{\alpha} \mu(x) \leqslant c\left(\int_{0}^{\infty}\left[t^{\alpha-N} \mu(B(x, t))\right]^{p^{\prime}} \frac{d t}{t}\right)^{1 / p^{\prime}}
$$

for some constant $c$ depending only on $\alpha, p$ and $N$, we get

$$
\mathscr{E}_{\alpha, p ; w}(\mu) \leqslant c \iint_{0}^{\infty}\left[t^{\alpha-N} \mu(B(x, t))\right]^{p^{\prime}} \frac{d t}{t} w(x)^{-1 / p-1} d x .
$$

Splitting off a factor of $\mu(B(x, t))$ in the above integral and changing the order of integration gives

$$
\int \mu(B(x, t))^{p^{\prime}} w(x)^{-1 / p-1} d x \leqslant \int \mu(B(y, 2 t))^{1 / p-1}\left(\int_{B(y, t)} w^{-1 / p-1}\right) d \mu(y) .
$$

Inserting this into (3.6) yields the desired upper bound on the energy. For the lower bound, we estimate $U_{\alpha, p ; w}^{\mu}$ from below and use (3.2). It always exceeds

$$
(N-\alpha) \int_{0}^{\infty} r^{\alpha-N}\left(\int_{|x-y|<r} w(y)^{-1 / p-1}\left[\int_{|x-z|<r}|y-z|^{\alpha-N} d \mu(z)\right]^{1 / p-1} d y\right) \frac{d r}{r}
$$

which exceeds

Hence

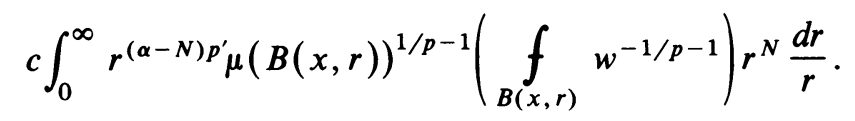

$$
U_{\alpha, p ; w}^{\mu}(x) \geqslant c W_{\alpha, p ; w}^{\mu}(x)
$$

for all $x$. 
4. Weighted Riesz capacity. In this section, we investigate the weighted Riesz capacity $R_{\alpha, p}^{w}(\cdot)$ defined by (2.6). To get at the various properties of these capacities needed in subsequent sections, we rewrite them in two ways, both times taking full advantage of the general theory of capacities corresponding to $L^{p}$-potentials as given by N. G. Meyers in [26]. Our objective is Theorem 4.1 below, a useful technical result that enables us to estimatee the weighted Riesz capacity of a set by getting upper and lower bounds for the $W$-potentials of measures concentrated on that set. It is from this result that sharp bounds for the weighted Riesz capacity of a ball are obtained in $\$ 6$. We begin with a short review of some of the main results from [26] slightly modified. The reader should have no trouble making the necessary alterations in the proofs given in [26].

Let $k(x, y)$ be a nonnegative, measurable function on $\mathbf{R}^{N} \times \mathbf{R}^{M}$. If $\mu$ and $\nu$ are Borel measures on $\mathbf{R}^{N}$ and $\mathbf{R}^{M}$ respectively, then we set $k(x, \mu)=\int k(x, y) d \mu(y)$, and similarly for $k(\nu, y)$ and $k(\nu, \mu)$. For $\nu$ fixed, we also assume that for all $f \geqslant 0$, $k(x, f \nu)$ is lower semicontinuous as a function of $x$. Here $f \nu$ is the measure $f(y) d \nu(y)$. A function $k$ with these properties will be referred to as a "kernel." Note that if $k$ is lower semicontinuous on $\mathbf{R}^{N} \times \mathbf{R}^{M}$ in addition to being nonnegative, then it is a kernel.

If $E$ is an arbitrary subset of $\mathbf{R}^{N}$, then its $L^{p}$-capacity with respect to the kernel $k$ is defined as

$$
C_{k ; \nu ; p}(E)=\inf \left\{\int f(y)^{p} d \nu(y): f \geqslant 0 \& k(x, f \nu) \geqslant 1, \forall x \in E\right\} .
$$

From the min-max theorem, it follows that for $E$ compact

$$
C_{k ; \nu ; p}(E)^{1 / p}=\sup \left\{\|\mu\|_{1}: \mu \in \mathscr{M}^{+}(E) \& \int k(\mu, y)^{p^{\prime}} d \nu(y) \leqslant 1\right\},
$$

where $\mathscr{M}^{+}(E)$ denotes those Borel measures supported on $E$ and $\|\mu\|_{1}$ is the total variation of $\mu$. Furthermore, for compact $E$, there exists a Borel measure $\mu_{E}$ supported on $E$ such that

$$
\begin{gathered}
\left\|\mu_{E}\right\|_{1}=C_{k ; \nu ; p}(E)=\int k\left(\mu_{E}, y\right)^{p^{\prime}} d \nu(y), \\
U^{\mu_{E}}(x) \equiv k\left(x, k\left(\mu_{E}, \cdot\right)^{1 / p-1} \nu\right) \geqslant 1 \quad \text { for } C_{k ; \nu ; p^{-a . e . ~} x \in E,} \\
U^{\mu_{E}}(x) \leqslant 1, \quad \forall x \in \operatorname{supp} \mu_{E} .
\end{gathered}
$$

The function $U^{\mu}$ defined in (4.4) is the nonlinear potential of the measure $\mu$ associated with the kernel $k$. For particular choices of $k$, the associated nonlinear potentials will correspond to the $U$-, $W$-potentials, respectively, of $\$ 2$.

From (4.3)-(4.5), one can easily deduce for compact $E$

$$
C_{k ; \nu ; p}(E)=\sup \left\{\|\mu\|_{1}: \mu \in \mathscr{M}^{+}(E) \& U^{\mu} \leqslant 1 \text { on } \operatorname{supp} \mu\right\} .
$$

Indeed, one writes

$$
\int k(\mu, y)^{p^{\prime}} d \nu(y)=\int U^{\mu}(x) d \mu(x)
$$


and

$$
\begin{aligned}
\mu(E) & \leqslant \int U^{\mu_{E}} d \mu \leqslant\|k(\mu, \cdot)\|_{L^{p^{\prime}}(\nu)}\left\|k\left(\mu_{E}, \cdot\right)\right\|_{L^{p^{\prime}}(\nu)}^{1 / p-1} \\
& \leqslant\|\mu\|_{1}^{1 / p^{\prime}}\left\|\mu_{E}\right\|_{1}^{1 / p} .
\end{aligned}
$$

Finally, we should note that $C_{k ; \nu ; p}(\cdot)$ is a monotone, countably subadditive outer set function (a capacity) on subsets of $\mathbf{R}^{N}$, and that all analytic sets are capacitible in the sense of Choquet.

Our first version of $R_{\alpha, p}^{w}$ in the Meyers format is obtained by simply setting $d \nu_{1}(y)=w(y) d y$ and $k_{1}(x, y)=|x-y|^{\alpha-N_{w}}(y)^{-1}$ in (4.1). Then for any $E \subset \mathbf{R}^{N}$,

$$
R_{\alpha, p}^{w}(E)=C_{k_{1} ; \nu_{1} ; p}(E)
$$

and it is easy to see that the nonlinear potentials associated with the kernel $k_{1}$ are just $U_{\alpha, p ; w}^{\mu}(x)$ of $\S 3$. Also due to (4.5) and (3.7),

$$
W_{\alpha, p ; w}^{\mu_{E}}(x) \leqslant c, \quad \forall x \in \operatorname{supp} \mu_{E}
$$

for some extremal measure associated with $k_{1}$.

Next, we find a comparable form for $R_{\alpha, p}^{w}$ using (3.4). Let $\chi(y)$ be the characteristic function of the open unit ball centered at the origin in $\mathbf{R}^{N}$, and set

$$
k_{2}(x,(y, t))=\chi((x-y) / t) t^{\alpha-N},
$$

for $t>0$, and zero otherwise. Here $x, y \in \mathbf{R}^{N}$ and now $M=N+1$ in (4.1). Also for the measure $\nu$ in (4.1), take $d \nu_{2}(y, t)=w(y)^{-1 / p-1} d y d t / t$, for $t>0$, and zero otherwise. The claim now is that

$$
R_{\alpha, p}^{w}(E) \sim C_{k_{2} ; \nu_{2} ; p}(E),
$$

for all sets $E$. To see this, note that the nonlinear potentials associated to the kernel $k_{2}$ are comparable to $W_{\alpha, p ; w}^{\mu}(x)$ of $\S 3$, hence by (3.2) and (3.4) we have for any $\mu$

$$
\left\|k_{1}(\mu, \cdot)\right\|_{L^{p^{\prime}}\left(\nu_{1}\right)} \sim\left\|k_{2}(\mu, \cdot)\right\|_{L^{p^{\prime}\left(\nu_{2}\right)}},
$$

which gives the claim because of (4.2). Also from (4.4) and (4.5), we get not only (4.8), but

$$
W_{\alpha, p ; w}^{\mu_{E}}(x) \geqslant 1 / c>0
$$

for $R_{\alpha, p}^{w}$-a.e. $x \in E$, for some extremal measure $\mu_{E}$ associated with $k_{2}$.

The following is a weighted analogue of Proposition 11 in [20]. The proof is a straightforward adaption of their proof.

Proposition 4.1. If $w$ satisfies (2.5), then for any $\mu \in \mathscr{M}^{+}$,

$$
R_{\alpha, p}^{w}\left(\left[W_{\alpha, p ; w}^{\mu}>\lambda\right]\right) \leqslant\left(c / \lambda^{p-1}\right)\|\mu\|_{1},
$$

with $c$ depending only on $\alpha, p, N, w$. 
Putting together (4.8), (4.11), (4.6) and (4.12) gives

THEOREM 4.2. Suppose $E$ is a compact set in $\mathbf{R}^{N}$, $\mu$ a Borel measure supported on $E$, and $w$ a weight satisfying (2.5).

(i) If $W_{\alpha, p ; w}^{\mu}(x) \leqslant 1, \forall x \in E$, then $R_{\alpha, p}^{w}(E) \geqslant c_{1}\|\mu\|_{1}$;

(ii) if $W_{\alpha, p ; w}^{\mu}(x) \geqslant 1, \forall x \in E$, then $R_{\alpha, p}^{w}(E) \leqslant c_{2}\|\mu\|_{1}$.

Here $c_{1}$ and $c_{2}$ depend only on $\alpha, p, N$ and $w$.

Finally, we remark on the necessity of condition (2.5). If $w_{\varepsilon}$ denotes $w$ truncated below by $\varepsilon>0$ (so $w_{\varepsilon} \geqslant \varepsilon$ ), and then in definition (2.6) we set $f(y)=A$. $w_{\varepsilon}(y)^{-1 / p-1}$ on $B(x, r)$, and zero otherwise, with $A=(2 r)^{N-\alpha}\left[w_{\varepsilon}^{-1 / p-1}(B(x, r))\right]^{-1}$, it follows that

$$
R_{\alpha, p}^{w}(B(x, r)) \leqslant c r^{N-\alpha p}\left(f_{B(x, r)} w^{-1 / p-1}\right)^{1-p} .
$$

In particular, if $R_{\alpha, p}^{w}$ is nontrivial, then $w^{-1 / p-1}$ must be locally integrable on $\mathbf{R}^{N}$. A similar argument gives that $|\log w|$ must be locally integrable also. Condition (4.14) is refined in $§ 6$. Note, however, that if

$$
w^{*}(x)=|x|^{N(p-1)}(\log 1 /|x|)^{p+\varepsilon-1}
$$

for $|x|<\frac{1}{2}$ and constant and continuous otherwise, then $w^{*}$ does not satisfy (2.5) if $\varepsilon>0$, though its weighted Riesz capacity is nontrivial. This last claim follows from the work of E. Sawyer [32] and will be commented on again in $\$ 7$ (Remark 7.2(b)).

\section{Weighted capacitary strong-type inequality.}

THEOREM 5.1. If $w$ satisfies (2.5), then there is a constant $c$ depending only on $\alpha, p$. $N$ and the $A_{\infty}$ constant such that

$$
\int_{0}^{\infty} R_{\alpha, p}^{w}\left(\left[I_{\alpha} * f \geqslant t\right]\right) d t^{p} \leqslant c \int f(x)^{p} w(x) d x,
$$

for all $f \geqslant 0$.

Estimate (5.1) is the capacitary strong-type inequality (CSI) corresponding to the weighted capacity $R_{\alpha, p}^{w}$. When the weight $w \equiv 1$, the proof of (5.1) has an extended history originating with V. G. Maz'ya in 1964. The best result of this type at present is due to K. Hansson [19] with a simplified proof by Maz'ya [24]. For a full discussion of these results, see [4] and Maz'ya's book [24]. The result of Hansson relies on the boundedness principle for nonlinear potentials associated with a convolution kernel: $k(x, y)=j(|x-y|)$ where $j(t)$ is generally assumed only to be nonincreasing in $t>0$. The principle roughly states that if the nonlinear potentials are bounded on the support of the measure, then they are bounded in all space. Unfortunately, there is no boundedness principle for the nonlinear potentials associated with the kernel $k_{1}$; in fact, these potentials can be finite on the support but infinite off the support - at a singularity of $w$. However, the good news is tiat the $W$-potentials, i.e. those associated with the kernel $k_{2}$, do satisfy this basic 
principle. This is easy to see for if $x \notin \operatorname{supp} \mu$, and if $x^{\prime}$ denotes a point of supp $\mu$ that minimizes the distance from $x$ to $\operatorname{supp} \mu$, then $\left|x^{\prime}-z\right| \leqslant 2|x-z|$ for all $z \in \operatorname{supp} \mu$. Hence $W^{\mu}(x) \leqslant c W^{\mu}\left(x^{\prime}\right)$.

Proof of TheOREM 5.1. (This proof is a modified version of Maz'ya's proof of Hansson's Theorem.) Let $J$ be the integral on the left side of (5.1). We may assume that $J$ is finite, for if not, we merely approximate $f$ by a sequence of bounded functions and the set $\left[I_{\alpha} * f \geqslant t\right]$ by a sequence of compact subsets and pass to the limit in the standard way using the limit theorems from [26]. With $\mu_{t}$ the measure of (4.3)-(4.5) for a compact subset of $N_{t}=\left[k_{1}\left(\cdot, f \nu_{1}\right) \geqslant t\right]$ associated with the kernel $k_{1}$, we have by (4.7)

$$
J \leqslant p \int_{0}^{\infty} \int k_{1}\left(x, f \nu_{1}\right) d \mu_{t}(x) t^{p-2} d t \leqslant p\|f\|_{L^{p}\left(\nu_{1}\right)} \cdot L^{1 / p^{\prime}}
$$

where

$$
L=\int\left[\int_{\mathrm{C}}^{\infty} k_{1}\left(\mu_{t}, y\right) t^{p-2} d t\right]^{p^{\prime}} w(y) d y .
$$

To conclude the proof, we simply show $L \leqslant c J$, for some constant $c$ depending only on the allowable parameters.

Case 1. $p \geqslant 2$. Letting $\lambda_{s}(E)=\int_{s}^{\infty} t^{p-2} \mu_{t}(E) d t$, we have by (3.6)

$$
\begin{aligned}
L & =\int\left[I_{\alpha} * \lambda_{0}(y)\right]^{p^{\prime}} w(y)^{-1 / p-1} d y \\
& \leqslant c \iint_{0}^{\infty}\left[r^{\alpha-N} \lambda_{0}(B(y, r))\right]^{p^{\prime}} \frac{d r}{r} w(y)^{-1 / p-1} d y .
\end{aligned}
$$

But

$$
\lambda_{0}(B(y, r))^{p^{\prime}}=p^{\prime} \int_{0}^{\infty} \lambda_{s}(B(y, s))^{p^{\prime}-1} \mu_{s}(B(y, r)) s^{p-2} d s
$$

and thus $L \leqslant c L_{1}^{2-p^{\prime}} L p^{p^{\prime}-1}$, where

$$
L_{1}=\iint_{0}^{\infty} \int_{0}^{\infty} r^{(\alpha-N) p^{\prime}} \mu_{s}(B(y, r))^{p^{\prime}} s^{p-1} d s \frac{d r}{r} w(y)^{-1 / p-1} d y
$$

and

$$
L_{2}=\iint_{0}^{\infty} \int_{0}^{\infty} r^{(\alpha-N) p^{\prime}} \mu_{s}(B(y, r))^{p^{\prime}} \lambda_{s}(B(y, r)) d s \frac{d r}{r} w(y)^{-1 / p-1} d y .
$$

We estimate $L_{1}$ by splitting off a factor of $\mu_{s}(B(y, r))$ and changing the order of integration as we did in the proof of Theorem 3.1. This leads to

$$
L_{1} \leqslant c \int_{0}^{\infty} s^{p-1} \int W_{\alpha, p ; w}^{\mu_{s}}(x) d \mu_{s}(x) d s \leqslant c \cdot J
$$

by (4.8) and the boundedness principle for the $W$-potentials. The same idea produces

$$
L_{2} \leqslant c \int_{0}^{\infty} \int_{s}^{\infty} t^{p-2} \int W_{\alpha, p ; w}^{\mu_{s}}(x) d \mu_{t}(x)^{\prime} d t d s \leqslant c \cdot J .
$$


Case 2. $1<p<2$. From (5.2), we write

$$
\lambda^{\infty}(B(y, r))^{p^{\prime}}=p^{\prime} \int_{0}^{\infty} \lambda^{s}(B(y, r))^{p^{\prime}-1} \mu_{s}(B(y, r)) s^{p-2} d s
$$

where

$$
\lambda^{s}(E)=\int_{0}^{s} t^{p-2} \mu_{t}(E) d t .
$$

Thus

$$
L \leqslant c \int_{0}^{\infty} s^{p-2}\left\|\lambda^{s}(B(\cdot, \cdot))\right\|_{L^{p^{\prime}-1}\left(\sigma_{s}\right)}^{p^{\prime}} d s
$$

where

$$
d \sigma_{s}(y, r)=r^{(\alpha-N) p^{\prime}} \mu_{s}(B(y, r)) w(y)^{-1 / p-1} d y \frac{d r}{r} .
$$

Note that

$$
\left\|\mu_{t}(B(\cdot, \cdot))\right\|_{L^{p^{\prime}-1}\left(\sigma_{s}\right)} \leqslant\left[\int W_{\alpha, p ; w}^{\mu_{t}} d \mu_{s}\right]^{p-1} \leqslant c\left\|\mu_{s}\right\|_{1}^{p-1} .
$$

Hence $L \leqslant c \cdot J$ in this case as well.

\section{Weighted Riesz capacity of a ball.}

THEOREM 6.1. (i) If $w$ satisfies (2.5), then there is a constant $c_{1}$ depending only on $\alpha$, $p, N$ and the $A_{\infty}$ constant such that

$$
R_{\alpha, p}^{w}(B(x, r)) \leqslant c_{1}\left[\int_{r}^{\infty} t^{(\alpha p-N) /(p-1)} \cdot f_{B(x, t)} w^{-1 / p-1} \cdot \frac{d t}{t}\right]^{1-p} ;
$$

(ii) if $w \in A_{p}$, then $R_{\alpha, p}^{w}(B(x, r))$ is comparable to the right side of (6.1).

When $w \equiv 1$ and $\alpha p<N$, estimate (6.1) reduces to the well-known estimate for the unweighted Riesz capacity of a ball; see [26]. Obviously, in order for (6.1) to be of any interest, we must assume that the integral in (6.1) is finite. If this is not the case for some $r>0$ and some $x$, then it is easy to see that actually $R_{\alpha, p}^{w}$ must be trivial. Alternate ways to proceed would be to either modify $w$ at infinity or to simply restrict attention to balls contained in a large fixed ball. It is the latter approach that was taken in [16] where, for $A_{2}$ weights, they derived part (ii) of our theorem using Green's function and the maximum principle for solutions of certain degenerate elliptic equations.

Proof of Theorem 6.1. In Theorem 4.2, we take $d \mu=A w(z) d z$ restricted to the ball $B(x, r), A$ a constant. Then splitting the range of integration in the integral defining $W_{\alpha, p ; w}^{\mu}(y)$, for $y \in B(x, r)$, into that over the intervals $(0,2 r)$ and $(2 r, \infty)$, we get for part (i) the lower estimate

$$
A^{1 / p-1} w(B(x, r))^{1 / p-1} \int_{2 r}^{\infty} t^{(\alpha p-N) /(p-1)} \cdot \int_{B(x, t / 2)} w^{-1 / p-1} \cdot \frac{d t}{t} .
$$

Choosing $A$ so that (6.2) is one, part (i) then follows by Theorem 4.2 and the doubling property of $A_{\infty}$ weights. To see (ii), we estimate the integral over $(2 r, \infty)$ from above in a similar fashion to $(6.2)$, whereas the integral over $(0,2 r)$ is bounded 
above by

$$
\int_{0}^{2 r} t^{\alpha p^{\prime}}\left(f_{B(y, t)} w\right)^{1 / p-1}\left(\underset{B(y, t)}{\left.f w^{-1 / p-1}\right) \frac{d t}{t}}\right.
$$

which does not exceed $c r^{\alpha p^{\prime}}$, which in turn does not exceed a quantity like (6.2). Hence the desired result again follows by Theorem 4.2 .

COROLlaRY 6.2. If $w$ satisfies (2.5), then there are constants $c_{1}$ and $c_{2}$ depending only on $\alpha, p, N$ and the $A_{\infty}$ constant such that

$$
\begin{aligned}
& R_{\alpha, p}^{w}(B(x, r)) \leqslant c_{1} r^{N-\alpha p}\left(f_{B(x, r)} w^{-1 / p-1}\right)^{1-p} \\
& \leqslant c_{2} r^{-\alpha p} w(B(x, r)) .
\end{aligned}
$$

Recall that (6.4) was obtained in (4.14) by a much less refined method.

COROLlaRY 6.3. (i) If $w$ satisfies (2.5), then there is a constant $c$ independent of $\sigma$ and $r$ such that for $0<\sigma \leqslant 1$,

$$
\left(\int_{r}^{\infty} t^{(\alpha p-N) /(p-1)} \cdot f_{B(x, t)} w^{-1 / p-1} \frac{d t}{t}\right)^{p-1} \cdot R_{\alpha, p}^{w}(B(x, \sigma r)) \leqslant c \sigma^{N-\alpha p-d},
$$

for any $d>d_{w}(x)$;

(ii) when $w \in A_{p}$, (6.6) becomes

$$
R_{\alpha, p}^{w}(B(x, \sigma r)) \leqslant c \cdot \sigma^{N-\alpha p-d} R_{\alpha, p}^{w}(B(x, r)),
$$

with the same qualifications as in (i).

EXAmPle 6.4. Let $w(x)=|x|^{\beta-N}$, then $w$ satisfies (2.5) when $\beta<N p$ and $A_{p}$ when $0<\beta<N p$. $d_{w}(0)=N-\beta$ and

$$
R_{\alpha, p}^{w}(B(0, r)) \sim \begin{cases}(\log 1 / r)^{1-p}, & \alpha p=\beta, \\ r^{\beta-\alpha p}, & \alpha p<\beta, \\ 1, & \alpha p>\beta,\end{cases}
$$

for $0<r<\frac{1}{2}$. Notice that (6.7) occurs at $x=0$ only when $\alpha p<N-d_{w}(0)$.

REMARKS 6.5. (a) If $w \in A_{p}$, then from Theorem 6.1 one has

$$
\frac{R_{\alpha, p}^{w}(B(x, r))}{R_{\alpha, p}(B(x, r))} \cdot(\underset{B(x, r)}{f w})^{-1} \sim 1
$$

as $r \rightarrow 0$, for a.e. $x$. Hence (6.5) can be reversed locally at least for a.e. $x$.

(b) Notice that if

$$
\int_{0}^{\infty} t^{(\alpha p-N) /(p-1)} \cdot f_{B(x, t)} w^{-1 / p-1}, . \frac{d t}{t}
$$


is finite, then $I_{\alpha} * f$ is continuous at $x$ when $w \in A_{p}$. In fact, (6.8) finite is equivalent to $R_{\alpha, p}^{w}(\{x\})>0$ and the estimate

$$
\left|I_{\alpha} * f(x+h)-I_{\alpha} * f(x)\right| \leqslant C R_{\alpha, p}^{w}(\{x\})^{-1 / p} \cdot\|f(\cdot+h)-f(\cdot)\|_{L^{p}(w)}
$$

holds.

\section{Two-weight strong-type estimates for Riesz potentials.}

THEOREM 7.1. Let $1<p<q<\infty, w$ satisfy (2.5), and $\mu$ a regular Borel measure on $\mathbf{R}^{N}$. Then the following are equivalent

(i) there is a constant $c_{1}$ such that

$$
\mu(B(x, r)) \leqslant c_{1} J_{x}(r)^{-q / p^{\prime}}
$$

for all $x \in \mathbf{R}^{N}$ and all $r>0$, where

$$
J_{x}(r)=\int_{r}^{\infty} t^{(\alpha p-N) /(p-1)} \cdot \int_{B(x, t)} w^{-1 / p-1} \cdot \frac{d t}{t} ;
$$

(ii) there is a constant $c_{2}$ such that

$$
\mu(B(x, r)) \leqslant c_{2} R_{\alpha, p}^{w}(B(x, r))^{q / p}
$$

for all $x \in \mathbf{R}^{N}$ and all $r>0$;

(iii) there is a constant $c_{3}$ such that

$$
\mu(K) \leqslant c_{3} R_{\alpha, p}^{w}(K)^{q / p}
$$

for all compact sets $K \subset \mathbf{R}^{N}$;

(iv) there is a constant $c_{4}$ such that

$$
\left\|I_{\alpha} * f\right\|_{L^{q}(\mu)} \leqslant c_{4}\|f\|_{L^{p}(w)}
$$

for all measurable $f \geqslant 0$.

Proof. The fact that (7.4) implies (7.3) implies (7.2) is trivial. Furthermore, it is clear that (6.1) yields that (7.2) implies (7.1) under assumption (2.5). Hence it remains to show that (7.1) implies (7.4). To this end, we obain the corresponding weak-type estimate and invoke the CSI (5.1). If we set $E_{\lambda}=\left[I_{\alpha} * f \geqslant \lambda\right]$ and let $\mu^{\lambda}$ be $\mu$ restricted to $E_{\lambda}$ (which we may assume to be compact), then we have

$$
\begin{aligned}
\lambda \mu\left(E_{\lambda}\right) & \leqslant \int f \cdot I_{\alpha} * \mu^{\lambda} d x \\
& \leqslant\|f\|_{L^{p}(w)}\left(\int\left(I_{\alpha} * \mu^{\lambda}\right)^{p^{\prime}} w^{-1 / p-1} d x\right)^{1 / p^{\prime}} .
\end{aligned}
$$

The last integral in the above expression is, of course, the energy of $\mu^{\lambda}, \mathscr{E}_{\alpha, p ; w}\left(\mu^{\lambda}\right)$, and is estimated by (3.4). That integral is then broken up in the usual way, part over the interval $(0, \sigma)$ and the rest over $(\sigma, \infty)$, with $\sigma$ to be specified. Each of these integrals is now estimated as follows:

$$
\begin{aligned}
\int_{0}^{\sigma} \cdots & \leqslant-c_{1}^{1 / p-1} \int_{0}^{\sigma} J_{x}(t)^{-q / p} d J_{x}(t) \\
& =c\left(J_{x}(\sigma)^{1-q / p}-J_{x}(0)^{1-q / p}\right),
\end{aligned}
$$


and

$$
\int_{\sigma}^{\infty} \cdots \leqslant \mu\left(E_{\lambda}\right)^{1 / p-1} \cdot J_{x}(\sigma)
$$

Now if $J_{x}(0)^{-q / p}<\mu\left(E_{\lambda}\right)^{1 / p-1}$, then we choose $\sigma=\sigma(x, \lambda)$ so that $J_{x}(\sigma)=$ $\mu\left(E_{\lambda}\right)^{-p^{\prime} / q}$, and if $J_{x}(0)^{-q / p} \geqslant \mu\left(E_{\lambda}\right)^{1 / p-1}$, then we set $\sigma=0$. In the first case, we have from (7.5)-(7.7) and (3.4),

$$
\lambda \mu\left(E_{\lambda}\right) \leqslant c\|f\|_{L^{p}(w)} \cdot \mu\left(E_{\lambda}\right)^{1-1 / q},
$$

which is the desired result. In the second case, (7.5) becomes

$$
\begin{aligned}
\lambda \mu\left(E_{\lambda}\right) & \leqslant c\|f\|_{L^{p}(w)}\left(\mu\left(E_{\lambda}\right)^{1 / p-1} \cdot \int J_{x}(0) d \mu^{\lambda}(x)\right)^{1 / p^{\prime}} \\
& \leqslant c\|f\|_{L^{p}(w)}\left(\mu\left(E_{\lambda}\right)^{1 / p-1-p^{\prime} / q+1}\right)^{1 / p^{\prime}}
\end{aligned}
$$

which again is the desired result.

Remarks 7.2. (a) In [32], E. Sawyer observes that once $w$ satisfies (2.5), then (7.4) is equivalent to

$$
\int_{Q}\left[I_{\alpha} * \mu_{Q}(x)\right]^{p^{\prime}} w(x)^{-1 / p-1} d x \leqslant c \mu(Q)^{p^{\prime} / q^{\prime}}<\infty
$$

holding for all cubes $Q$ in $\mathbf{R}^{N}, 1<p \leqslant q<\infty$. Here $\mu_{Q}$ denotes $\mu$ restricted to the cube $Q$. It should be noted, however, that although (7.1) is somewhat simpler than (7.8), (7.8) remains equivalent to (7.4) when $q=p$, whereas (7.1) is not. For the unweighted case and condition (7.1), see [1 and 2].

(b) Actually, the main result of [32] is that (7.8) is equivalent to a $\left(L^{q}(\mu), L^{p}(w)\right)$ weak-type estimate analogous to the $\left(L^{q}(\mu), L^{p}(w)\right)$ strong-type estimate (7.4) in Theorem 7.1, for arbitrary $w \geqslant 0$. And furthermore, the example $w^{*}$ at the end of $\S 4$ satisfies (7.8) for $\varepsilon \leqslant(p-1) /(q-1)$ and

$$
d \mu(x)=|x|^{q(\alpha-N)-N}(\log 1 /|x|)^{1 / q-1} d x
$$

for $|x|<\frac{1}{2}$ and zero otherwise, whereas (7.4) fails with $f(x) \sim w^{*}(x)^{-1 / p-1}$ in $|x|<\frac{1}{2}$.

(c) From [18], one has that if $w \in A_{p}$ and $d \mu(x)=v(x) d x$, with $v$ a doubling weight, and for all cubes $Q$

$$
v(Q)^{1 / q}|Q|^{\alpha / N} \leqslant c w(Q)^{1 / p}
$$

then (7.4) holds. Note that (7.9) is a consequence of (7.2) and (6.5), though our $\mu$ in (7.4) need not be a doubling measure.

(d) Finally, we remark that it is possible to obtain limiting results (as would be expected as $q \rightarrow \infty$ ) in the spirit of N. Trudinger [35] by adapting the methods of [3] to the present setup. In fact, if $w$ satisfies (2.5) and if

$$
\Psi(t)=\sup \int_{0}^{1} J_{x}\left(t^{1 / d} s\right) s^{d / p-1} \frac{d s}{s}
$$


where the supremum is taken over all $x \in \operatorname{supp} \mu$, then $\mu(B(x, t)) \leqslant c t^{d}$, for all $x$ and all $t>0$, and some fixed $d>0$, implies

$$
\int_{B} \Phi\left(I_{\alpha} * f\right) d \mu \leqslant \int_{0}^{\infty} \Psi^{-1}\left(c \lambda^{p^{\prime}}\right) d \Phi(\lambda)
$$

for all $f \geqslant 0$ with support contained in a fixed ball $B$. Here $c$ is a constant independent of $f$, if $\|f\|_{L^{p}(w)} \leqslant 1$. Also $\Psi^{-1}$ is the essential inverse of $\Psi$ and $\Phi$ is any nondecreasing function with $\Phi(0)=0$. For example, if $t^{-\alpha p} w(B(x, t)) \leqslant$ $(\log 1 / t)^{-\gamma}$, as $t \rightarrow 0$, then (7.10) implies that there are constants $c$ and $\beta$ independent of $f$ such that

$$
\int_{B} \exp \left[\beta\left(I_{\alpha} * f\right)^{q}\right] d \mu \leqslant c
$$

with $q=p /(p-1+\gamma)$ and $\|f\|_{L^{p}(w)} \leqslant 1$. The case $\gamma=0, \mu=$ Lebesgue $N$-measure is the result of Trudinger, whereas if $\gamma=0$ with $\mu$ as above, see [3].

When $q=p,(7.3)$ and (7.4) are still equivalent due to CSI for weights satisfying (2.5). And as we have pointed out, (7.1) is now no longer equivalent to (7.4). A condition depending only on balls or cubes equivalent to (7.4) is, of course, E. Sawyer's condition (7.8). However, recently another sufficient condition for (7.4) was discovered by C. Fefferman [17] while investigating the Schrödinger operators. Extensions of his result have also been given in [12, 6, and 13]. We present here a two-weight version of the result in [6].

THEOREM 7.3. If $w \in A_{p}, 1<p<\infty$, then there is a constant $c$ depending only on $\alpha, p, N, s$ and the $A_{p}$ constant of $w$ such that

$$
\int\left(I_{\alpha} * f\right)^{p} v d x \leqslant c \int f^{p} \cdot N_{s} \cdot w d x
$$

for all $f \geqslant 0$. Here $v \geqslant 0$ and

$$
N_{s}(x)=\sup _{Q \ni x}|Q|^{\alpha p / N}\left(f_{Q} w\right)^{-1}\left(f_{Q} v(y)^{s} d y\right)^{1 / s},
$$

$s>1$, and $Q$ a coordinate cube.

The proof of this result relies on the methods of obtaining estimates for fractional maximal functions due to E. Sawyer [31]. In particular, we need

LEMmA 7.4. If $M_{\beta}^{w} \mu(x)=\sup _{Q \ni x}|Q|^{\beta / N} \cdot w(Q)^{-1} \cdot \mu(Q)$ with $w \in A_{p}, 1<p<$ $\infty$, then

$$
\int\left(M_{\alpha}^{1} f\right)^{p} d \mu \leqslant c \int f^{p} \cdot M_{\alpha p}^{w} \mu \cdot w d x
$$

for all $f \geqslant 0$. Here $c$ is a constant depending on $\alpha, p, N$ and the $A_{p}$ constant of $w$.

This lemma follows as in [4, p. 22f], where Sawyer's approach is slightly modified yielding (7.14) for weight $w \equiv 1$. The idea is to use the covering lemma of Carleson [11]; see also [22]. We give here an outline of the argument. 


$$
\begin{aligned}
\int_{0}^{\infty} \mu\left[M_{\alpha}^{1} f>t\right] d t^{p} & \leqslant c \sum_{k} 2^{k p} \mu\left[2^{k}<M_{\alpha}^{1} f \leqslant 2^{k+1}\right] \\
& \leqslant c \sum_{k, j} 2^{k p} \mu\left(Q_{j}^{k}\right) \leqslant c \sum_{k, j} \mu\left(Q_{j}^{k}\right)\left|Q_{j}^{k}\right|^{\alpha p / N}\left(f_{Q_{j}^{k}} f\right)^{p}
\end{aligned}
$$

where $\left\{Q_{j}^{k}\right\}$ is a cover of a sufficiently large bounded portion $G_{k}$ of $\left[2^{k}<M_{\alpha}^{1} f \leqslant\right.$ $2^{k+1}$ ] consisting of coordinate cubes $Q$ centered at $x$ for which

$$
\frac{1}{2} M_{\alpha}^{1} f(x) \leqslant|Q|^{\alpha / N-1} \int_{Q} f
$$

$x \in G_{k}$. Thus

$$
\int\left(M_{\alpha}^{1} f\right)^{p} d \mu \leqslant c \sum_{k, j}\left[f_{Q_{j}^{k}} f \cdot\left(M_{\alpha p}^{w} \mu\right)^{1 / p} d x\right]^{p} w\left(Q_{j}^{k}\right) .
$$

If the $Q_{j}^{k}$ 's were disjoint, then the lemma would be immediate from Hölder's inequality. They are not disjoint however, but Sawyer has shown that they can be chosen so that they satisfy the Carleson condition

$$
\sum_{Q_{j}^{k} \subset 4 Q_{s}^{t}} w\left(Q_{j}^{k}\right) \leqslant c \cdot w\left(Q_{s}^{t}\right)
$$

for all pairs $(s, t)$; see [31]. Thus from [22], the right side of (7.15) is bounded by the right side of (7.14) which gives the lemma.

ProOF OF THEOREM 7.3.

$$
\begin{aligned}
\int\left(I_{\alpha} * f\right)^{p} v d x & \leqslant \int\left(I_{\alpha} * f\right)^{p}\left[M_{0}^{1} v^{a}\right]^{1 / a} d x \\
& \leqslant c \int\left(M_{\alpha}^{1} f\right)^{p}\left[M_{0}^{1} v^{a}\right]^{1 / a} d x \leqslant c \int f^{p} M_{\alpha p}^{w}\left(\left[M_{0}^{1} v^{a}\right]^{1 / a}\right) w d x
\end{aligned}
$$

The second inequality in (7.16) follows from Proposition 3.1 since by [15], $\left[M_{0}^{1} v^{a}\right]^{1 / a}$ is an $A_{1}$ weight and hence an $A_{\infty}$ weight, for any $a>1$. The third inequality of (7.16) is just Lemma 7.4. Thus it remains to show that

$$
M_{\alpha p}^{w}\left(\left[M_{0}^{1} v^{a}\right]^{1 / a}\right) \leqslant c \cdot N_{s}
$$

for $1<a<s$. This is easy: split $v=v_{1}+v_{2}, v_{1}=v$ restricted to $B(x, 2 r)$, then

$$
\int_{B(x, r)}\left[M_{0}^{1} v_{1}^{a}\right]^{s / a} d y \leqslant c \int_{B(x, 2 r)} v^{s} d y
$$

and for $y \in B(x, r)$

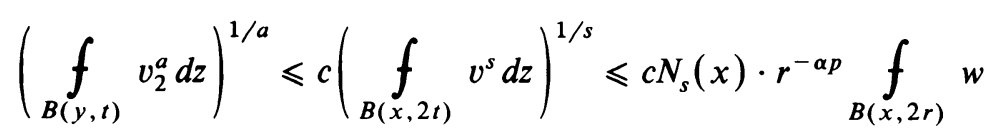

by (2.4) since now $r \leqslant t$. 
Two-weight first-order strong-type for weights not satisfying (2.5). A form of weighted capacity more closely associated with second-order quasi-linear elliptic partial differential equations is, for $E$ compact,

$$
C_{p}^{w}(E)=\inf \int|\nabla \phi(x)|^{p} w(x) d x
$$

where the infimum is over all $\phi \in C_{0}^{\infty}\left(\mathbf{R}^{N}\right)$ for which $\phi \geqslant 1$ on $E$. Here $\nabla \phi$ denotes the usual gradient vector of $\phi$; see [16 and 34]. Since the estimate $|\phi(x)| \leqslant$ $c \cdot I_{1} *|\nabla \phi|(x)$ is elementary, it follows that

$$
R_{1, p}^{w}(E) \leqslant c \cdot C_{p}^{w}(E)
$$

holds for all weights and all compact sets. However, the reverse of (7.17) generally only holds for $A_{p}$ weights. Thus when $w^{-1 / p-1} \notin L_{\mathrm{loc}}^{1}, R_{1, p}^{w} \equiv 0$ (see (4.14)), but $C_{p}^{w}(\cdot)$ may well be nontrivial. This last fact may be deduced, for example, using the results of [18], in particular, they show

Proposition 7.6. If $1<p<q<\infty, w(x)=|x|^{p \beta}, v(x)=|x|^{q \gamma}$, then

$$
\|\phi\|_{L^{q}(v)} \leqslant c\|\nabla \phi\|_{L^{p}(w)}
$$

for all $\phi \in C_{0}^{\infty}\left(\mathbf{R}^{N}\right)$-with support disjoint from the origin when $v \notin L_{\mathrm{loc}}^{1}-$ iff $1 / q+$ $\gamma / N=1 / p+(\beta-1) / N \neq 0$ and $1 / p-1 / q \leqslant 1 / N$.

In fact, it follows from this that for $w(x)=|x|^{\beta-N}, 1<p<\beta, C_{p}^{w}(B(0, r)) \sim$ $r^{\beta-p}$, for all $r>0$. However, what is more interesting is that Proposition 7.6, and various extensions of it, can be deduced directly from Theorem 7.1 by a transformation of coordinates.

COROllaRY 7.7. Let $y=f(x)$ be a diffeomorphism of $\mathbf{R}^{N}$ onto itself and $w$ a weight for which $\tilde{w} \in A_{p}, \tilde{w}(y)=w \circ f^{-1}(y) \cdot\left|f^{-1 \prime}(y)\right|^{1-p / N}$ with $\left|f^{-1 \prime}(y)\right|$ the absolute value of the Jacobian for the inverse $x=f^{-1}(y)$. Suppose further that for all balls $B$

$$
\int_{f^{-1}(B)} v(x) d x \leqslant c^{\prime} \cdot C_{p}^{w}\left(f^{-1}(B)\right)^{q / p}
$$

for $1<p<q<\infty$ and some constant $c^{\prime}$ independent of $B$, then (7.18) holds for some constant $c$ independent of $\phi \in C_{0}^{\infty}\left(\mathbf{R}^{N}\right)$.

The corollary follows immediately from Theorem 7.1, due to the fact that now $R_{1, p}^{\tilde{w}}(\cdot) \sim C_{p}^{\tilde{\omega}}(\cdot)$ and $C_{p}^{\tilde{\omega}}(B)=C_{p}^{w}\left(f^{-1}(B)\right)$ and $\int_{B} v \circ f^{-1}(y) \cdot\left|f^{-1^{\prime}}(y)\right| d y=$ $v\left(f^{-1}(B)\right)$.

For Proposition 7.6 in the case $\gamma q+N>0$, apply the corollary to $f(x)=x|x|^{\alpha}$, $\alpha>\max \{-1,(\beta p+N-N p) / p(N-1)\}$, and notice that $f^{-1}(B)$ is comparable to a ball $B^{\prime}$. In fact, for small balls centered away from the origin, it is necessary to have $1 / p-1 / q \leqslant 1 / N$, while for large balls or balls centered at the origin, the other restriction on the exponents comes into play. And finally, Proposition 7.6 for $\gamma q+N<0$ follows by inverting the result just established via $T x=x \cdot|x|^{-2}$.

REMARK 7.8. From (7.8) with $p \leqslant q$ and $\alpha=1$ one can deduce via (7.3) and (7.17) that (7.18) holds for all $\phi \in C_{0}^{\infty}\left(\mathbf{R}^{N}\right)$ when $v \in L_{\text {loc }}^{1}$-see [5, Theorem 2]-but that (7.4) may fail with $\alpha=1$ as pointed out in Remark 7.2(b). 
8. Weighted thinness. Let $(w, v)$ be a pair of weights satisfying (2.5). A subset $E \subset \mathbf{R}^{N}$ will be termed $(\alpha, p)$-thin at $x$ with respect to the pair $(w, v)$ if

$$
\int_{0}^{1}\left[t^{\alpha p-N} R_{\alpha, p}^{w}(E \cap B(x, t))\right]^{1 / p-1}\left(f_{B(x, t)} v^{-1 / p-1}\right) \frac{d t}{t}<\infty
$$

whenever

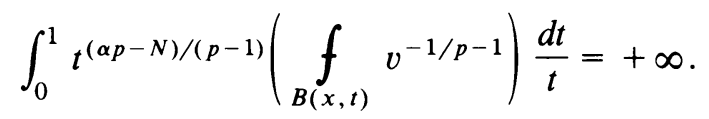

The main purpose of this section is to formulate conditions on the pairs $(w, v)$ and $(\bar{w}, \bar{v})$ such that $(\alpha, p)$-thinness at $x$ with respect to the first pair coincides with $(\alpha, p)$-thinness at $x$ with respect to the second pair. Note that when both weights $w$ and $v$ are identically one, then (8.1) reduces to the unweighted $(\alpha, p)$-thinness of the literature-see esp. [27, 20,7]-and when $\alpha=1, p=2$, this unweighted thinness coincides with the classical notion due to Brelot [10]. Also, when $\alpha=1, p=2$, and $w=v,(8.1)$ and (8.2) agree with the notion of thinness (irregularity) introduced in [16] for boundary regularity of solutions to degenerate elliptic second-order divergence-form partial differential equations. The present definition is motivated by the above results as well as those of Stredulinsky [34] and Bauman [9]. Our main observation is Theorem 8.2. We first need some preliminaries.

Lemma 8.1. Let $w$ and $\bar{w}$ satisfy (2.5). For $k \in \mathbf{Z}^{+}$and $0<\rho<1$, set

$$
A_{k}(x, \rho)=\left\{y: \rho^{k+1} \leqslant|x-y|<\rho^{k}\right\}
$$

and

$$
A_{k}^{*}(x, \rho)=\left\{y: \rho^{k+j} \leqslant|x-y|<\rho^{k-1}\right\}
$$

for some fixed $j \in \mathbf{Z}^{+}, j>1$. Then there exists a $\rho_{0} \in(0,1]$, independent of $k$ and the set $E$ such that

$$
R_{\alpha, p}^{w}\left(E \cap A_{k}(x, \rho)\right) \leqslant 2^{p}\left[\sup _{A_{k}^{*}} \frac{w}{\bar{w}}\right] \cdot R_{\alpha, p}^{\bar{w}}\left(E \cap A_{k}(x, \rho)\right)
$$

for all $\boldsymbol{\rho} \in\left(0, \rho_{0}\right]$ and all $k \in \mathbf{Z}^{+}$, provided

$$
\alpha p<N-d_{\bar{w}}(x) .
$$

PROOF. Let $f$ be the capacitary extremal function for $R_{\alpha, p}^{\bar{w}}\left(E \cap A_{k}(x, \rho)\right)$, and set

$$
f_{k}(y)= \begin{cases}f(y), & \rho^{k+j} \leqslant|x-y|<\rho^{k-1}, \\ 0, & \text { otherwise. }\end{cases}
$$

We will pick $\rho_{0}$ so that $I_{\alpha} * f_{k}(z) \geqslant \frac{1}{2}$ for all $z \in E \cap A_{k}(x, \rho)$. First write

$$
\begin{gathered}
I_{\alpha} * f_{k}(z) \geqslant 1-\int_{|y-x|<\rho^{k+j}} \cdots-\int_{|y-x| \geqslant \rho^{k-1}} \cdots \\
=1-I_{1}-I_{2} . \\
I_{1} \leqslant c \rho^{(k+1)(\alpha-N)} \int_{|y-x|<\rho^{k+j}} f(y) \bar{w}(y)^{1 / p} \cdot \bar{w}(y)^{-1 / p} d y \\
\leqslant c \rho^{(k+1)(\alpha-N)}\|f\|_{L^{p}(\bar{w})} \cdot\left(\int_{|y-x|<\rho^{k+j}} \bar{w}(y)^{-1 / p-1} d y\right)^{1 / p^{\prime}} .
\end{gathered}
$$


And with (2.4) and (6.4), we get

$$
I_{1} \leqslant c \rho^{\alpha-N+N j \delta / p^{\prime}}
$$

Thus we choose $j>(N-\alpha) p^{\prime} / N \delta$ so that $I_{1}<\frac{1}{4}$ for all $\rho \leqslant \rho_{0}$.

$$
\begin{aligned}
I_{2} & \leqslant c\left(\int_{|y-x| \geqslant \rho^{k-1}}|z-y|^{(\alpha-N) p^{\prime}} \bar{w}(y)^{-1 / p-1} d y\right)^{1 / p^{\prime}} \cdot\|f\|_{L^{p}(\bar{w})} \\
& \leqslant c\left(\int_{\rho^{k-1}}^{\infty} t^{(\alpha p-N) /(p-1)} \cdot \int_{B(x, t)} \bar{w}^{-1 / p-1} \cdot \frac{d t}{t}\right)^{1 / p^{\prime}} \cdot R_{\alpha, p}^{\bar{w}}\left(B\left(x, \rho^{k}\right)\right)^{1 / p}
\end{aligned}
$$

which, according to (6.6), does not exceed $c \rho^{(N-\alpha p-d) / p}$. Thus we again restrict $\rho_{0}$ so that $I_{2}<\frac{1}{4}$ for all $\rho \leqslant \rho_{0}$. The lemma now easily follows from

$$
\begin{aligned}
R_{\alpha, p}^{w}\left(E \cap A_{k}(x, \rho)\right) & \leqslant 2^{p} \int f_{k}(y)^{p} w(y) d y \\
& \leqslant 2^{p}\left(\sup _{A_{k}^{*}} \frac{w}{\bar{w}}\right) \cdot \int f(y)^{p} \bar{w}(y) d y .
\end{aligned}
$$

The condition we impose on the pairs of weights is the following one: there is a constant $c$ independent of $k$ such that for each fixed $\rho \in(0,1)$,

$$
\sup _{A_{k}(x, \rho)} \frac{w}{\bar{w}} \leqslant c \cdot \inf _{A_{k}(x, \rho)} \frac{v}{\bar{v}}
$$

for all $k \in \mathbf{Z}^{+}$. It might be of interest to notice that when $v=w, \bar{v}=\bar{w},(8.5)$ is an equivalence relation between $w$ and $\bar{w}$.

THEOREM 8.2. If the pairs $(w, v)$ and $(\bar{w}, \bar{v})$ satisfy (2.5), (8.2) and (8.5), then $(\alpha, p)$-thinness at $x$ with respect to $(w, v)$ coincides with $(\alpha, p)$-thinness at $x$ with respect to $(\bar{w}, \bar{v})$, when

$$
\alpha p<N-\max \left(d_{w}(x), d_{v}(x), d_{\bar{w}}(x), d_{\bar{v}}(x)\right) .
$$

COROLlaRY 8.3. If the weight $w$ satisfies (2.5), (8.2) and

$$
\sup _{A_{k}(x, \rho)} w \leqslant c \inf _{A_{k}(x, \rho)} w
$$

for all $k \in \mathbf{Z}^{+}, c$ independent of $k$, then $(\alpha, p)$-thinness at $x$ with respect to $(w, w)$ coincides with unweighted $(\alpha, p)$-thinness at $x$, when $\alpha p<N-\max \left(d_{w}(x), 0\right)$.

Proof of Theorem 8.2. From Lemma 8.1 the idea is clear: write the integral in (8.1) in terms of rings and apply (8.3) and (8.5). For this we need

LEMMA 8.4. There is a constant $c>0$ such that

$$
\begin{aligned}
& \sum_{k=0}^{\infty}\left[R_{\alpha, p}^{w}\right.\left.\left(E \cap B\left(x, \rho^{k}\right)\right) \rho^{k(\alpha p-N)}\right]^{1 / p-1} \cdot f_{B\left(x, \rho^{k}\right)} v^{-1 / p-1} \\
& \leqslant c \sum_{k=0}^{\infty}\left[R_{\alpha, p}^{w}\left(E \cap A_{k}(x, \rho)\right) \rho^{k(\alpha p-N)}\right]^{1 / p-1} \cdot f_{B\left(x, \rho^{k}\right)} v^{-1 / p-1},
\end{aligned}
$$

$1<\rho<\infty, \alpha p<N-d_{v}(x)$, for all $\rho \leqslant \rho_{0}$. 
Proof. Since

$$
R^{w}\left(E \cap B\left(\rho^{k}\right)\right)^{1 / p-1} \leqslant c_{p} R^{w}\left(E \cap A_{k}\right)^{1 / p-1}+c_{p} R^{w}\left(E \cap B\left(\rho^{k+1}\right)\right)^{1 / p-1}
$$

with $c_{p}=1$ for $p \geqslant 2$, we form the sum on the left side of (8.7) using (8.8). The result then follows upon invoking property (2.4) for the weight $v^{-1 / p-1}$ and changing the summation index in the last sum. The result is that the last sum then contains a factor of $\rho$ raised to a power larger than $\left(N-\alpha p-d_{v}(x)\right) /(p-1)$. Hence one need only restrict $\rho \leqslant \rho_{0}$ for some small $\rho_{0}$.

Thus to complete the proof of Theorem 8.2, we observe that the integral in (8.1) for the pair $(w, v)$ does not exceed a constant multiple of the left side of (8.7), and the right side of (8.7), together with (2.4) and (8.3), does not exceed a multiple of

$$
\sum_{k}\left[R^{\bar{w}}\left(E \cap A_{k}\right) \rho^{k(\alpha p-N)}\right]^{1 / p-1} \cdot f_{B\left(\rho^{k}\right)} \bar{v}^{-1 / p-1} \cdot E_{k}
$$

where

$$
E_{k}=\left(\min _{m} \inf _{A_{m}} \frac{v}{\bar{v}}\right)^{-1 / p-1} \cdot\left(\sup _{A_{k}^{*}} \frac{w}{\bar{w}}\right)^{1 / p-1}
$$

with $m$ an integer from the set $\{k-1, \ldots, k-1+j\}$. Now choose $A_{m}$ to be that ring of $A_{k}^{*}$ where the supremum of $w / \bar{w}$ is assumed. Thus by (8.5), $E_{k}$ is bounded independently of $k$, and one half of the theorem is established. For the other half, merely interchange the roles of $(w, v)$ and $(\bar{w}, \bar{v})$.

EXAMPLE 8.5. (a) If $w(x)=|x|^{\beta-N}, 0<\beta<N p$, then it is easy to check Corollary 8.3 to see that the two notions of weighted and unweighted $(\alpha, p)$-thinness at the origin coincide when $\alpha p<\min (N, \beta)$, and differ when $\beta \leqslant \alpha p \leqslant N$.

If $\beta \geqslant N p$, then $w$ no longer satisfies (2.5). In this case we must modify our definition of thinness slightly. We use the capacities $C_{p}^{w}$ of $\S 7$, and say that the set $E$ is $p$-thin at $x$ with respect to the weight $w$-now at least $L_{\text {loc }}^{1}$-if

$$
\int_{0}^{1}\left[\frac{t^{p} C_{p}^{w}(E \cap B(x, t))}{w(B(x, t))}\right]^{1 / p-1} \frac{d t}{t}<\infty
$$

whenever

$$
\int_{0}^{1}\left[\frac{t^{p}}{w(B(x, t))}\right]^{1 / p-1} \frac{d t}{t}=+\infty .
$$

Notice that (8.10) and (8.11) agree with (8.1) and (8.2) when $v=w \in A_{p}$. Also $p$-thinness with respect to weight $w$ agrees with the corresponding notions of irregularity in [16, 34, and 9].

Returning to our example, we see that if $f(x)=x|x|^{\alpha}, \alpha>-1$, and, as before, $\tilde{w}(y)=w \circ f^{-1}(y) \cdot\left|f^{-1^{\prime}}(y)\right|^{1-p / N}$, then it is always possible to choose $\alpha$ so that both $\tilde{w} \in A_{p}$ and $d_{\tilde{w}}(0)<0$ happen when $\beta \geqslant N p$. And (8.10) is comparable to

$$
\int_{0}^{1}\left[\frac{t^{p} C_{p}^{\tilde{w}}(f(E) \cap B(0, t))}{\tilde{w}(B(0, t))}\right]^{1 / p-1} \frac{d t}{t}
$$


which in turn is comparable to the transformed unweighted version of (8.12) (with $\tilde{w}$ for $\beta=N)$ when $p<N-\alpha(N-p) /(1+\alpha)$ i.e. $p<N$. Thus weighted and unweighted $p$-thinness at the origin for the weight $w(x)=|x|^{\beta-N}, \beta>0$, are the same provided $p<\min (\beta, N)$.

(b) If $N=3, w(x, y, z)=\left(x^{2}+y^{2}\right)^{\delta / 2}$, and $E=\left\{(0,0, z): 0 \leqslant z \leqslant \frac{1}{2}\right\}$, then $E$ is not $(1,2)$-thin when $-1<\delta<0$, but is $(1,2)$-thin when $\delta=0$.

(c) If $N=3, w(x, y, z)$ as in (b), and $E=\left\{(\rho, z): \rho \leqslant z^{2} \leqslant \frac{1}{2}\right\}$, then $E$ is $(1,2)$-thin for $0<\delta<1$, but not $(1,2)$-thin when $\delta=0$.

(d) If $N=3, w(x, y, z)=|z|^{\delta}$, and $E$ is a Cantor set in the $(x, y)$-plane of Hausdorff dimension $1+\varepsilon, 0<\varepsilon<\delta<1$, then $R_{1,2}^{w}(E)=0$, but $R_{1,2}(E)>0$. Thus not only are weighted and unweighted thin points different in general, they are not even the same up to a set of capacity zero.

The remaining two examples concern the boundary regularity for the Dirichlet problem for certain second-order elliptic partial differential equations.

(e) Consider the equation

$$
\Delta u+\alpha(|x|)\left(x /|x|^{2}\right) \cdot \nabla u=0
$$

in a bounded domain $\Omega \subset \mathbf{R}^{N}$ with the origin on the boundary of $\Omega$. Here $\alpha(t)$ is a bounded measurable function on $0 \leqslant t<\infty$. It has been known for some time now that if $\alpha(|x|) \cdot|x|^{-1} \in L_{\text {loc }}^{N}$ on $\mathbf{R}^{N}$, then the origin is a regular point for (8.13) iff it is a regular point for the Laplace equation $\Delta u=0$ in $\Omega$; see [33]. Recently in [16], we learned to rewrite (8.13) as

$$
\frac{\partial}{\partial x_{j}}\left(w(x) u_{x_{j}}\right)=0 \quad \text { (summation convention) }
$$

where $w(x)=\exp -\int_{|x|}^{R} \alpha(t) d t / t$, when $|x|<R$, and one otherwise. Here $R=$ $\operatorname{diam} \Omega$. Also from [16], we know that the origin is a regular point for (8.14) in $\Omega$ iff $\Omega^{c}=$ the complement of $\Omega$ is not 2-thin at 0 with respect to the weight $w(x)$. But if $-\alpha_{0} \leqslant \alpha(t) \leqslant \alpha_{1}, \alpha_{0}, \alpha_{1} \geqslant 0$, then Corollary 8.3 and the techniques of Corollary 7.7 imply that the origin is a regular point for (8.14) in $\Omega$ iff it is a regular point for Laplace's equation in $\Omega$, when $\alpha_{0}<N-2$. Thus, in particular, this will always be the case if $N>2$ and $\lim _{t \rightarrow 0} \alpha(t)=0$ by suitably localizing the Dirichlet problem.

(f) Finally, we consider the equation

$$
a_{i j}(x) u_{x_{i} x_{j}}=0 \quad \text { (summation convention) }
$$

again in $\Omega$ with $0 \in \partial \Omega$, where $a_{i j}(x)=\delta_{i j}+\beta(|x|) x_{i} x_{j} /|x|^{2}$, and $\beta(t)=$ $-\alpha(t) /(N-1+\alpha(t)), \alpha(t)$ as in (e). From [9], we have that the origin is irregular for (8.15) in $\Omega$ iff $\Omega^{c}$ is 2-thin at 0 with the weight $g\left(x_{0}, x\right)$, the Green's function for (8.15) in a large fixed ball containing $\Omega$ with pole at $x_{0} \notin \bar{\Omega}$. Furthermore, Bauman shows that in this case

$$
\int_{r}^{1} \frac{t^{2}}{w(B(0, t))} \frac{d t}{t} \sim \int_{r}^{1} t^{2-N} \exp \int_{t}^{1} \alpha(s) \frac{d s}{s} \cdot \frac{d t}{t} .
$$


Thus upon integrating by parts twice,

$$
\int_{0}^{1} \frac{t^{2} C_{2}^{w}(E \cap B(0, t))}{w(B(0, t))} \frac{d t}{t} \sim \int_{0}^{1} t^{2-N} C_{2}^{w}(E \cap B(0, t)) \cdot \exp \int_{t}^{1} \alpha(s) \frac{d s}{s} \cdot \frac{d t}{t}
$$

since

LEMMA 8.6. For any doubling weight $w$,

$$
C_{p}^{w}(B(0, r)) \leqslant c\left[\int_{r}^{\infty}\left(\frac{t^{p}}{w(B(0, t))}\right)^{1 / p-1} \frac{d t}{t}\right]^{1-p},
$$

for some constant $c$ independent of $r ; 1<p<\infty$.

Thus 2-thinness of $\Omega^{c}$ at 0 with respect to the weight $g\left(x_{0}, x\right)$ is equivalent to 2-thinness of $\Omega^{c}$ at 0 with weight $\exp -\int_{|x|}^{1} \alpha(s) d s / s$. Consequently, the origin is a regular point for (8.13) iff it is regular for (8.15), and when $\alpha_{0}<N-2$, iff it is regular for Laplace's equation.

Proof of Lemma 8.6. Set

$$
\phi(x)= \begin{cases}\int_{|x|}^{\infty}\left(\frac{t^{p}}{\omega(t)}\right)^{1 / p-1} \frac{d t}{t}, & |x| \geqslant r, \\ \phi(r), & |x|<r,\end{cases}
$$

where $\omega(t)=w(B(0, t))$. Then if we set $f(t)=t^{p^{\prime}} \omega(t)^{-p^{\prime}}$, we have

$$
\int|\nabla \phi(x)|^{p} w(x) d x \leqslant \int_{r}^{\infty} f(\rho) d \omega(\rho) \leqslant c \cdot \phi(r)
$$

since $w$ is a doubling weight. Upon normalizing, the result follows.

\section{REFERENCES}

1. D. R. Adams, Traces of potentials arising from translation invariant operators, Ann. Scuola Norm. Sup. Pisa 25 (1971), 203-217.

2. __ A trace inequality for generalized potentials, Studia Math. 48 (1973), 99-105.

3. __ Traces of potentials. II, Indiana Univ. Math. J. 22 (1973), 907-918.

4. __ Lectures on $L^{p}$-potential theory, Umeå Univ. Reports, No. 2, 1981.

5. __ Capacity and the obstacle problem, Appl. Math. Optim. 8 (1981), 39-57.

6. Some weighted estimates for potentials, Abstracts Amer. Math. Soc. 5 (1984), 355.

7. D. R. Adams and N. G. Meyers, Thinness and Wiener criteria for nonlinear potentials, Indiana Univ. Math. J. 22 (1972), 169-197.

8. Bessel potentials. Inclusion relations among classes of exceptional sets, Indiana Univ. Math. J. 22 (1973), 873-905.

9. P. Bauman, A Wiener test for nondivergence structure second-order elliptic equations, preprint.

10. M. Brelot, Sur les ensembles effilés, Bull. Sci. Math. 68 (1944), 12-36.

11. L. Carleson, An interpolation problem for bounded analytic functions, Amer. J. Math. 80 (1958), 921-930.

12. S. Y. A. Chang, J. M. Wilson and T. H. Wolff, Some weighted norm inequalities concerning the Schrödinger operators, preprint.

13. S. Chanillo and $\mathrm{R}$. Wheeden, $L^{p}$ estimates for fractional integrals and Sobolev inequalities with applications to Schrödinger operators, preprint.

14. R. Coifman and C. Fefferman, Weighted norm inequalities for maximal functions and singular integrals, Studia Math. 51 (1974), 241-250. 
15. R. Coifman and R. Rochberg, Another characterization of BMO, Proc. Amer. Math. Soc. 79 (1980), 249-254.

16. E. Fabes, D. Jerison and C. Kenig, The Wiener test for degenerate elliptic equations, Ann. Inst. Fourier (Grenoble) 32 (1982), 151-182.

17. C. Fefferman, The uncertainty principle, Bull. Amer. Math. Soc. (N.S.) 9 (1983), 129-206.

18. A. Gatto, C. Gutiérrez and R. Wheeden, Fractional integrals on weighted $H^{p}$ spaces, Trans. Amer. Math. Soc. 289 (1985), 575-589.

19. K. Hansson, Imbedding theorems of Sobolev type in potential theory, Math. Scand. 45 (1979), 77-102.

20. L. I. Hedberg and T. H. Wolff, Thin sets in nonlinear potential theory, Ann. Inst. Fourier (Grenoble) 33 (1983), 161-187.

21. Nonlinear potentials and approximation in the mean by analytic functions, Math. Z. 129 (1972), 299-319.

22. L. Hormander, $L^{p}$ estimates for ( pluri-) subharmonic functions, Math. Scand. 20 (1967), 65-78.

23. S. V. Hruščev, A description of weights satisfying the $A_{\infty}$ condition of Muckenhoupt, Proc. Amer. Math. Soc. 90 (1984), 253-257.

24. W. Maz'ja, Zur theorie Sobolewscher Räume, Teubner-Texte sur Math., Band 38, Leipzig, 1981.

25. W. Maz'ja and V. Havin, Nonlinear potential theory, Russian Math. Surveys 27 (1972), 71-148.

26. N. G. Meyers, A theory of capacities for potentials of functions in Lebesgue spaces, Math. Scand. 26 (1970), 255-292.

27. Continuity properties of potentials, Duke Math. J. 42 (1975), 157-166.

28. B. Muckenhoupt, Weighted norm inequalities for the Hardy maximal function, Trans. Amer. Math. Soc. 165 (1972), 207-226.

29. The equivalence of two conditions for weight functions, Studia Math. 49 (1974), 101-106.

30. B. Muckenhoupt and R. Wheeden, Weighted norm inequalities for fractional integrals, Trans. Amer. Math. Soc. 192 (1974), 261-274.

31. E. Sawyer, A characterization of a two weight norm inequality for maximal operators, Studia Math. 75 (1982), 1-11.

32. A two weight weak type inequality for fractional integrals, Trans. Amer. Math. Soc. 281 (1984), 339-345.

33. G. Stampacchia, Le probléme de Dirichlet pour les équations elliptiques du second ordre à coefficients discontinus, Ann. Inst. Fourier (Grenoble) 15 (1965), 189-258.

34. E. Stredulinsky, Weighted inequalities and degenerate elliptic partial differential equations, Lecture Notes in Math., vol. 1074, Springer-Verlag, Berlin and New York, 1984.

35. N. Trudinger, On imbeddings into Orlicz spaces and some applications, J. Math. Mech. 17 (1967), $473-483$.

Department of Mathematics, University of Kentucky, Lexington, Kentucky 40506 